\title{
Experimental and analytical studies to substantiate the blasting operations rational parameters in the hard media destruction
}

\author{
Ihor Kratkovskyi ${ }^{1, *}$, and Oleksii Ishchenko ${ }^{2}$ \\ ${ }^{1}$ Institute of Geotechnical Mechanics named by N. Poljakov of National Academy of Sciences of \\ Ukraine, 49005, Dnipro, Simferopolska Str., 2a, Ukraine \\ ${ }^{2}$ National TU Dnipro Polytechnic, 49005, Dnipro, Dmytra Yavornytskoho Ave., 19, Ukraine
}

\begin{abstract}
The method of sieve analysis studied particle size distribution of the products explosive destruction of sand-cement models with elliptical compensatory cavity in its middle that were destroyed with use of various designs explosive charges and built the cumulative distribution curves. It was established that the area of the newly formed surface of these models destruction products, destroyed by an explosion of variable cross section charges, increased 1.45 times as compared with charges of other structures (boiler in the extension of the charge end, solid structure). The middle diameter of particles increased at 50-60\%. According to the results of the fractional composition, distribution of the products models destruction for different designs and geometric parameters of the compensation cavity has been defined by the regression lines. According to the cumulative distribution curves of the products of destruction of a model with different geometrical parameters of the compensation cavity, around which various designs of explosive charges were exploded, the equations of the regression lines was determined. Based on the obtained dependences of the regression-correlation analysis, mathematical models have been developed to optimize the choice of rational parameters of compensation cavities and explosive charge structures. Recommendations for their use in mines are given.
\end{abstract}

\section{Introduction}

Preparation and carrying mining various purposes drilling and blasting method is one of the most important components of the process. At this stage, the efficiency of mining with the use of explosion energy is determined.

Selection rational technology, which provides a high-speed drifting preparatory workings is essential intensification of mining operations [1-2].

In this regard, the drifting efficiency can be improved by solving a number of tasks, including both improving the organization of labor and improving the parameters of drilling and blasting operations [3-4].

${ }^{*}$ Corresponding author: kratkovsky@i.ua 
One of the ways to improve the efficiency of drilling and blasting operations in the destruction of hard rock of complex structure is to take into account the rock massif structure, physic mechanical properties, as well as blasting parameters and the design of explosive charges [5]. The purpose of the research is to substantiate the design of the explosive charge and parameters of the compensation cavity in order to increase the efficiency of hard media destruction.

\section{Methods of experimental studies of the solid hard destruction by the explosion}

Numerous studies have established that the nature of the rocks destruction by an explosion depends on their physical and mechanical properties, the mining and geological mineral deposits conditions, as well as on the parameters of the explosive impulse. When extracting a certain part of the rock mass by an explosion with its good conditional crushing, movement and formation of the compact broken-rock-pile, to ensure the subsequent efficient operation of the technological equipment, it is necessary to have a free surface with sufficient volume.

Such conditions are characteristic of underground mines, when the carrying out of horizontal and vertical mine workings in viscous stressed hard rock's of a complex structure is conducted in a state of "clamping" from the rock pressure forces action.

Crushing of the character massif explosion greatly influenced by changing the depth increases the development of physical and mechanical properties of the rock, and small cross sectional area openings.

When this destruction and rock release from the borehole charge is carried out of the funnel, which in section becomes elliptical in shape [6-7].

In order to substantiate the parameters of conducting preparatory workings method [8-10] and to determine the features of the hard medium state in the "clamping" mode and the explosive impulse parameters of acting on the charging cavity surface from the charge explosion of various designs to the destruction rock in the landfill conditions were carried out the experimental studies. In this case, sand-cement models were used.

According to the developed method of modelling [11], and research methodology models produced in the form of cylinders with a diameter of $270 \mathrm{~mm}$ and a height of $200 \mathrm{~mm}$.

Simulation of a stressed state of the environment was created by forming a sand-cement mixture in a steel form, which had an outer cylindrical shell with a fixing device for its position. The shape of the model was made of sheet steel with a wall thickness of 2-3 mm. The sand-cement mixture was prepared in proportion: quartz sand + cement grade $400=1: 1$ with addition of $10 \%$ water. In the process of manufacturing, a model in the center of it to create an additional free surface in the sand and cement mixture set the insert to form a compensatory cavity of the elliptical shape. Geometric elliptical insert the following: the ellipsoid major axis $40 \mathrm{~mm}$, oriented in the direction of possible rock pressure and low axis $30 \mathrm{~mm}$. The insert in the model was set to a depth of $180 \mathrm{~mm}$ (Fig. 1). Around the compensation cavity from the center of a circle with radius $R=(0.3$ 0.35) $d_{\text {mod }}$ vertices inscribed in a square shaped inserts using four cylindrical cavity for containing the explosive charge. Inserts with a diameter of $10 \mathrm{~mm}$ were placed at a depth of $170 \mathrm{~mm}$. The scheme of the model is shown in Figure 2.

Simultaneously with the manufacture of basic models, samples were made to determine the density $\rho$, the velocity of $c_{p}$-waves and the strength of uniaxial compression of models $\sigma_{\text {comp }}$ in accordance with the current State standards [12-16]. 


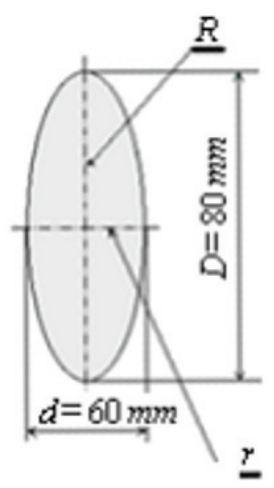

Fig. 1. Appearance and parameters of the compensation cavity: $R$ is large and $r$ small an ellipse semiaxis.
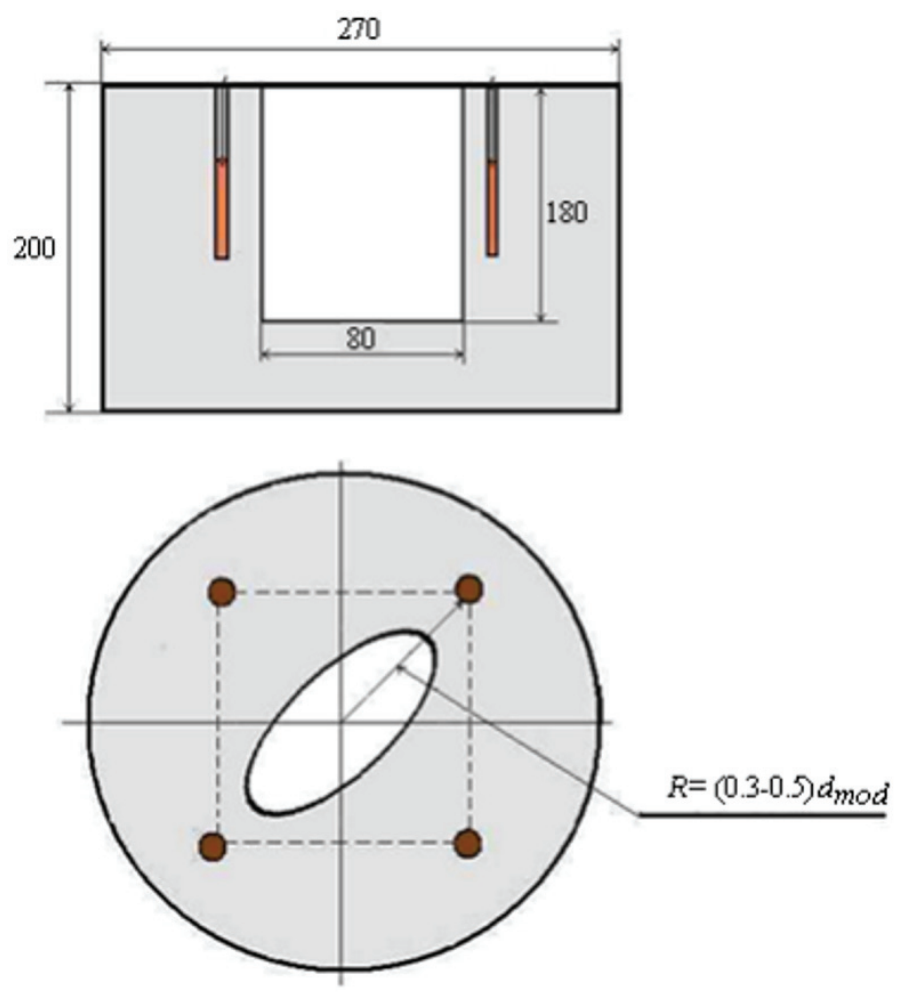

Fig. 2. Scheme cylinder model with a set of holes around the cavity compensation.

After a set maximum strength, models were conducted experimental research in landfill conditions Flux-Dolomite Combine (Dokuchaievsk). In explosive cavities, prepared models shaped elongated explosive charge different designs: a permanent section with boiler expansion in the end borehole, with a spherical end inserted into the borehole, spherical inserts (Fig. 3). The explosive was placed in a cartridge of cylindrical shape with a diameter of 0.9-0.95 from the diameter of the explosive cavity, made of parchment. As a blast, a mixture was used which, in its characteristics, is close to industrial explosive (for example, Gramonite 79/21), in the following ratio: pentolite $(80 \%)$ and solid rocket fuel $(20 \%)[17]$. 


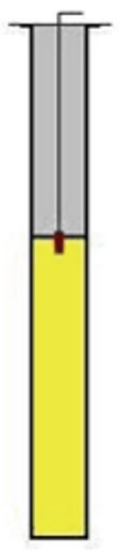

$a$
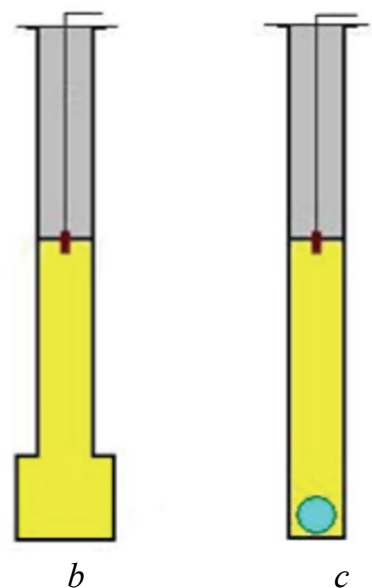

$c$

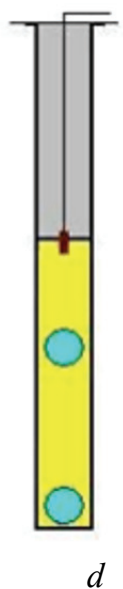

Fig. 3. Explosive charge designs: $a$-charge with constant section; $b$-boiler charge of expansion in the bit-end fault; $c$ - charge with a spherical insertion in the end hole; $d$ - charge with spherical inserts.

For bursting of charges, the initiators were made in the form of a cylindrical shape cartridge with a diameter of 5-6 mm in which placed $80-100 \mathrm{mg}$ of high-explosive substance with an incandescence bridge. The charges were sealed with a $0.25 \mathrm{~mm}$ fraction quartz sand stemming. The total mass of the explosive in charges was $4.0 \mathrm{~g}$, the blasting ratio $-0.33 \mathrm{~kg} / \mathrm{m}^{3}$. The charges in the model were exchanged with the network of the explosive device [18]. The model was installed in a metal box (explosive chamber) and remotely undermined from the shelter (Fig. 4) with a delay, which began with a pair of charges located in the possible direction zone of the prevailing forces action of the massif pressure on compensating cavity, namely, along the larger axis an elliptical cavity, and a pair of other charges - along the line perpendicular to the line of its smaller axis.

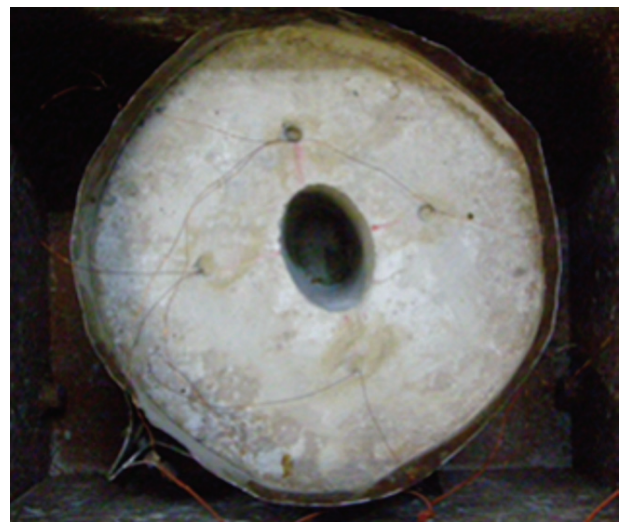

$a$

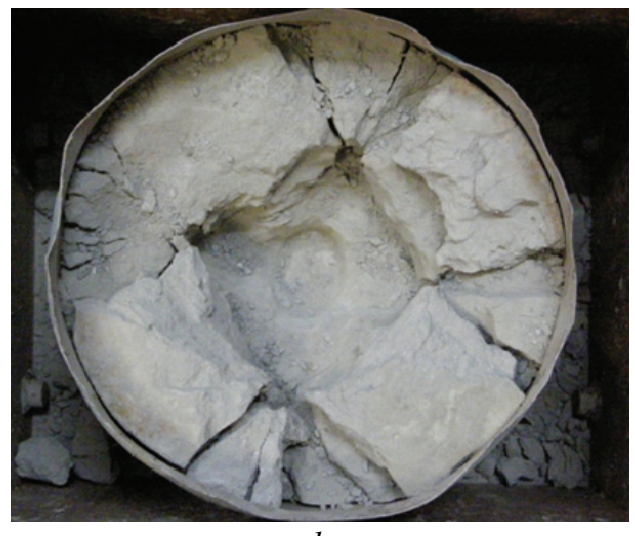

$b$

Fig. 4. General view of a cylindrical model in an explosive chamber: $a$ - before the explosion; $b-$ after the explosion.

After each explosion by sieve analysis using laboratory studies conducted size, distribution of the destruction models products. To do this, a set of sieves with apertures of 0.25 in size was used; $0.5 ; 1.0 ; 2.0 ; 3.0 ; 5.0 ; 7.0 ; 10.0 ; 12.0 ; 16.0 ; 20.0 ; 26.0 ; 30.0 ; 40.0$; $50.0 ; 60.0 ; 70.0 ; 80.0 \mathrm{~mm}$. 
The study size distribution models destroyed by approved methods [19] calculated the average diameter of the piece, the newly created surface area, volume shattered models (Table 1). Then they built cumulative curves (Fig. 5).

Table 1. Results of crushing sand-cement models with compensating cumulative explosives charges of different constructions.

\begin{tabular}{|c|c|c|c|c|c|c|}
\hline \multirow[t]{2}{*}{$\begin{array}{c}\text { Constructi } \\
\text { ons of } \\
\text { charges }\end{array}$} & \multirow{2}{*}{$\begin{array}{l}\text { The mass } \\
\text { of the } \\
\text { model } \\
\text { blown up } \\
\text { by } \\
\text { explosion } \\
m, \mathrm{~kg}\end{array}$} & \multirow{2}{*}{$\begin{array}{c}\text { Diameter } \\
\text { of middle } \\
\text { piece } \\
d_{\text {mid }}, \mathrm{mm}\end{array}$} & \multicolumn{2}{|c|}{$\begin{array}{l}\text { Composition of } \\
\text { fractions }(\%) \\
\text { destroyed by } \\
\text { explosion of the } \\
\text { model }\end{array}$} & \multirow{2}{*}{$\begin{array}{l}\text { The volume } \\
\text { of the model } \\
\text { blown up by } \\
\text { explosion } \\
V \cdot 10^{-3}, \mathrm{~m}^{3}\end{array}$} & \multirow{2}{*}{$\begin{array}{l}\text { Newly } \\
\text { formed } \\
\text { surface } \\
S_{n .}, \mathrm{cm}^{2}\end{array}$} \\
\hline & & & $\begin{array}{c}d_{i}<20 \\
\mathrm{~mm}\end{array}$ & $\begin{array}{c}d_{i}>50 \\
\mathrm{~mm}\end{array}$ & & \\
\hline $\begin{array}{l}\text { Charge of } \\
\text { constant } \\
\text { section }\end{array}$ & 1.270 & 22.55 & 51.45 & 20.0 & 0.66 & 32548 \\
\hline $\begin{array}{l}\text { Charge } \\
\text { with } \\
\text { spherical } \\
\text { insertion } \\
\text { at the end } \\
\text { of the hole }\end{array}$ & 3.965 & 33.53 & 44.1 & 29.4 & 2.5 & 72670 \\
\hline $\begin{array}{l}\text { Charge } \\
\text { with } \\
\text { evenly } \\
\text { spaced } \\
\text { columns } \\
\text { with } \\
\text { spherical } \\
\text { inserts }\end{array}$ & 3.525 & 37.7 & 50.0 & 26.4 & 2.86 & 78600 \\
\hline $\begin{array}{l}\text { Charge } \\
\text { with boiler } \\
\text { expansion } \\
\text { in the end }\end{array}$ & 2.100 & 23.3 & 51.1 & 19.6 & 1.09 & 62088 \\
\hline
\end{tabular}

Analysis of the results showed fragmentation patterns (Table 1), that the newly created surface area destroyed by explosion explosive charge variable section compared to charges of other designs (with boiler expansion in the end charge, solid construction) increased by 1.4-1.45 times, and the diameter of the middle piece is 50-60\%. The uniform distribution of granulometric composition for charges of variable cross-section is established in comparison with other structures of charges (Fig. 5). In this case, for charges of a variable cross-section evenly distributed output, both small and large fractions, increases the average piece and mass of the blown-up part of the model.

\section{Mathematical modeling study and choice of rational parameters of blasting effectively destroying rocks}

During testing, it was found that the number of different types fractions depending on the 
form of compensation cavity in the model center. During the research, the following data were recorded: size of the fraction, average fraction size, mass of the $i$-th fraction, part of the $i$-th fraction in the total mass of the destroyed part of the model, the average diameter of the $i$-th fraction, the area of the newly formed surface of the $i$-th fraction, the total area newly formed surface. For solving the problem, regression models were constructed that reflect the relationship between fractions of different types and the ratio of the axes of the ellipse. Based on mathematical models has been developed optimizing choice of parameters rational compensational cavity and type explosive charge design. The chosen optimization criterion is the maximum yield of the average fractions [20]. Investigation of the correlation dependence between the main indicators (the size of the fraction, the average fraction size, the mass of the $i$-th fraction, the fraction of the $i$-th fraction in the total mass of the destroyed part of the model, the average diameter of the $i$-th fraction, the area of the newly formed surface of the $i$-th fraction, the total area of the newly formed surface) characterizing the explosion showed that these parameters are connected by a rather linear correlation bond, since the correlation coefficients are within $[0.7 ; 1]$. Therefore, in the subsequent study for the main parameter one of them was chosen, namely, part of the second fraction in the total mass of the destroyed part of the model.

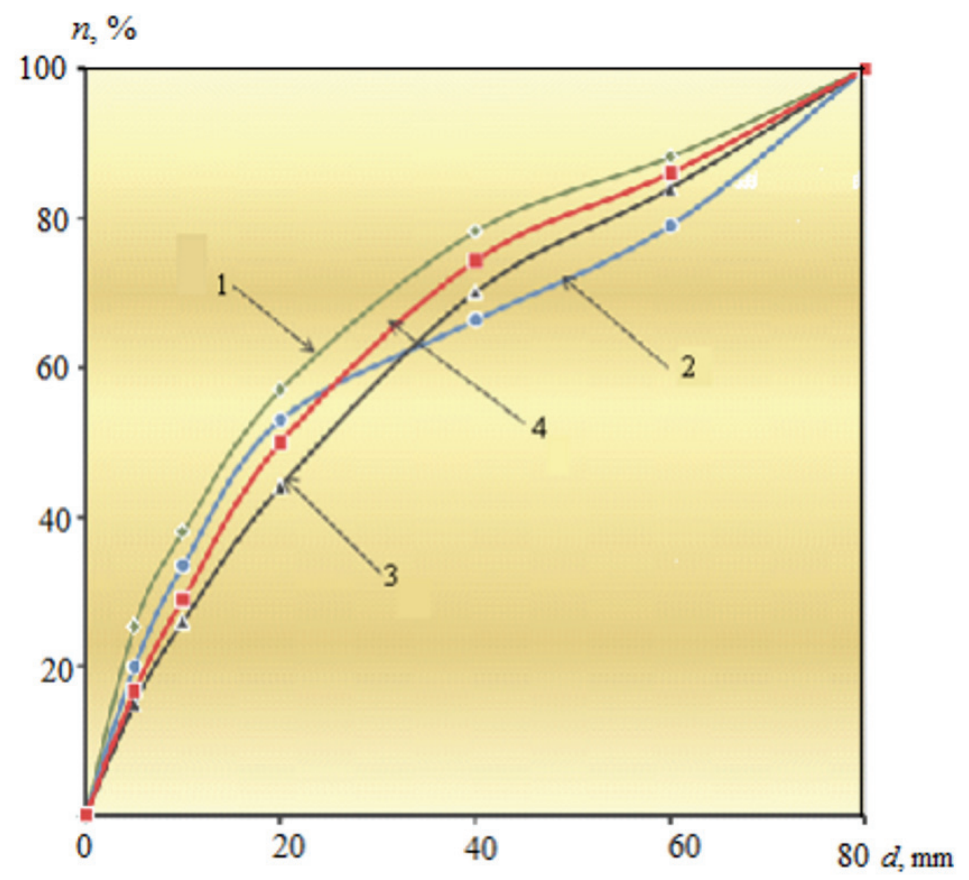

Fig. 5. Dependence of the faction splitting of size distribution in the particle size distribution of the explosion shattered the model of compensation cavity explosive charge different designs: 1, 2, 3, 4 charges of explosive substance of constant section; with boiler expansion at the end of the hole; with spherical insertion at the end of the hole; with spherical inserts placed evenly over the column.

In this regard, we investigate the dependence of the fractional composition on the parameters of the compensating cavity and construct the corresponding regression equations.

For example, let's consider the results of 16 experiments in which the smaller axis of the ellipse varies from 45 to $75 \mathrm{~mm}$, and larger - from $65 \mathrm{~mm}$ to $95 \mathrm{~mm}$. Data obtained as a result of the experiment on the destruction of models by charges of exploding solid construction are given in Table 2. 
Table 2. Distribution of factions splitting patterns in the destruction of the explosive charge constant cross section of resizing compensation elliptical cavity.

\begin{tabular}{|c|c|c|c|c|c|c|}
\hline $\begin{array}{l}\text { Experiment } \\
\text { number }\end{array}$ & $\begin{array}{l}\text { Small } \\
\text { axis } d_{1}\end{array}$ & $\begin{array}{c}\text { Big axis } \\
d_{2}\end{array}$ & $\begin{array}{l}\text { Axis } \\
\text { ratio, } X\end{array}$ & $\begin{array}{l}\text { Number of } \\
\text { small } \\
\text { fractions, } \%\end{array}$ & $\begin{array}{l}\text { Number of } \\
\text { average } \\
\text { fractions, } \%\end{array}$ & $\begin{array}{l}\text { Number of } \\
\text { large } \\
\text { fractions, } \%\end{array}$ \\
\hline 1 & 45 & 65 & 0.692 & 48.6 & 21.3 & 30.0 \\
\hline 2 & 47 & 67 & 0.701 & 49.4 & 22.5 & 28.1 \\
\hline 3 & 49 & 69 & 0.710 & 50.5 & 22.5 & 27.0 \\
\hline 4 & 51 & 71 & 0.718 & 51.8 & 22.6 & 25.6 \\
\hline 5 & 53 & 73 & 0.726 & 52.6 & 23,0 & 24.4 \\
\hline 6 & 55 & 75 & 0.733 & 53.3 & 22.9 & 23.7 \\
\hline 7 & 57 & 77 & 0.740 & 54.1 & 23.1 & 22.8 \\
\hline 8 & 59 & 79 & 0.747 & 55.0 & 23.3 & 21.7 \\
\hline 9 & 61 & 81 & 0.753 & 55.7 & 23.2 & 21.0 \\
\hline 10 & 63 & 83 & 0.759 & 56.7 & 22.9 & 20.4 \\
\hline 11 & 65 & 85 & 0.765 & 57.2 & 23 & 19.8 \\
\hline 12 & 67 & 87 & 0.770 & 58.1 & 22.5 & 19.4 \\
\hline 13 & 69 & 89 & 0.775 & 58.7 & 22.8 & 18.5 \\
\hline 14 & 71 & 91 & 0.780 & 59.4 & 23.0 & 17.6 \\
\hline 15 & 73 & 93 & 0,785 & 60,1 & 22.9 & 17.0 \\
\hline 16 & 75 & 95 & 0.789 & 61.2 & 22.4 & 16.4 \\
\hline
\end{tabular}

Similar results are obtained in explosive destruction of models by charges of explosive substances of variable section, as well as with a boiler expansion at the end of the hole, with spherical insertion at the end and with spherical inserts that alternate evenly along the column of charge. There has been investigated dependence of the number of small, medium and large fractions of the form of compensation cavity. To do this, the correlation coefficients were calculated and a correlation relationship was established between the parameters under study. The results of calculations are given in Tables 3 and 4.

Since the absolute value of the correlation coefficient number of small and large diameters factions and compensation empty near to 1 , it can be concluded that the values associated linear relationship. The correlation between the diameters of the cavity and the number of middle fractions is small, but the correlation ratio is within the permissible 
limits, which suggests a nonlinear dependence between these parameters.

Table 3. Linear correlation distribution of fractional composition shattered explosion models charge the permanent section on the parameters of the compensation cavity elliptical shape.

\begin{tabular}{|c|c|c|}
\hline Indexes & $\begin{array}{c}\text { Less axis of compensating } \\
\text { cavity }\end{array}$ & $\begin{array}{c}\text { Large axis of the } \\
\text { compensating cavity }\end{array}$ \\
\hline Number of small fractions & 0.92871 & 0.92871 \\
\hline $\begin{array}{c}\text { Number of average } \\
\text { fractions }\end{array}$ & 0.67082 & 0.67082 \\
\hline Number of large fractions & 0.93095 & 0.93095 \\
\hline
\end{tabular}

Table 4. Non-linear correlation distribution of fractional composition shattered explosion models charge the permanent section on the parameters of the compensation cavity elliptical shape.

\begin{tabular}{|c|c|c|}
\hline Indexes & $\begin{array}{c}\text { Less axis of compensating } \\
\text { cavity }\end{array}$ & $\begin{array}{c}\text { Large axis of the } \\
\text { compensating cavity }\end{array}$ \\
\hline Number of small fractions & 0.9976 & 0.99761 \\
\hline $\begin{array}{c}\text { Number of average } \\
\text { fractions }\end{array}$ & 0.3766 & 0.37655 \\
\hline Number of large fractions & -0.988 & -0.9882 \\
\hline
\end{tabular}

The experimental data distribution fractional composition devastated parts of the model, such as continuous charge compensation structure and parameters cavity elliptical shape depending on the regression line (Fig. 6), described by the equations of regressioncorrelation analysis.

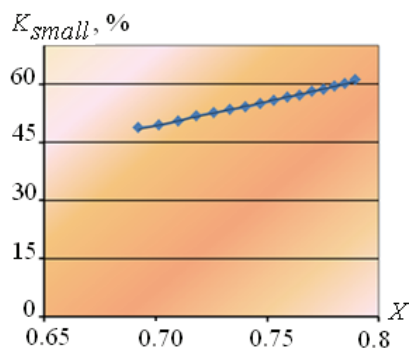

$a$
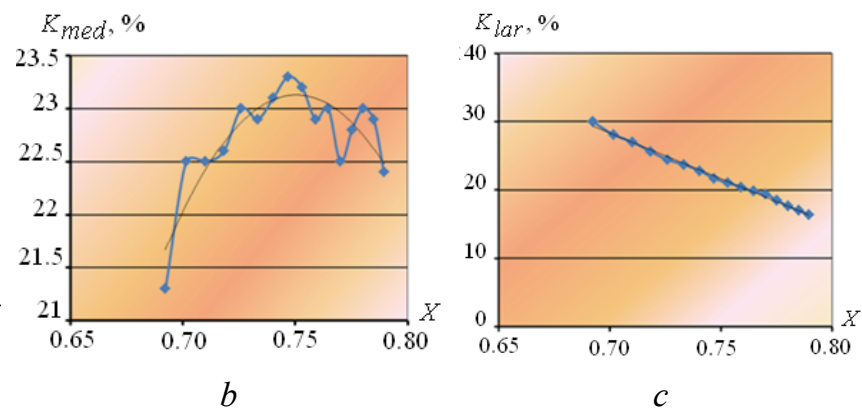

Fig. 6. The dependence of changes in the number of factions splitting ratio of compensation cavity axis elliptical shape model destroyed explosive charge constant section: $a$ - the number of shallow fractions, $\% ; X=d_{1} / d_{2}$ - the ratio of the axes of the ellipse, the regression equation: $K_{\text {small }}=126.3 X-39.15$; correlation coefficient $R^{2}=0.9964 ; b-$ number of average fractions, $\%,(X<1)$, regression equation: $K_{\text {med }}=-429.8 X^{2}+642.5 X-219.0 R^{2}=0.943 ; c$ - number of large fractions, \%, regression equation: $K_{\text {lar }}=-133.3 X+121.6 ; R^{2}=0.9952$.

From the graphs, it is seen that the relation between the number of small and large fractions and the diameters of the compensatory cavity of the elliptic form has a linear dependence, while the insignificant deviation of the regression line from the input data is estimated by the coefficient of approximation, which tends to 1 . That is, the assumption of linear dependence is fair. Linear dependence is observed also between the number of large fractions and the ratio of the axis of the cavity. The corresponding calculation for the 
number of middle fractions confirms that the relationship is not linear.

Consider a mathematical model 1 . We introduce the notation: $d_{1}-$ small axis; $d_{2}-$ large axis of the compensatory cavity of the elliptical form.

Then a mathematical model with one unknown:

$$
K_{\text {small }}\left(d_{1} / d_{2}\right) \rightarrow \max
$$

namely:

$$
-429.8\left(d_{1} / d_{2}\right)^{2}+645.2\left(d_{1} / d_{2}\right)-219 \rightarrow \max
$$

under conditions:

$$
\begin{gathered}
K_{\text {small }}+K_{\text {med }}+K_{\text {lar }} \leq 100 ; \\
K_{\text {small }} \geq K_{\text {small.pr }} ; \\
K_{\text {lar }} \leq K_{\text {lar.pr }} \\
K_{\text {lar }} \geq 0 .
\end{gathered}
$$

Then:

$$
\begin{gathered}
K_{\text {med }}=126.3\left(d_{1} / d_{2}\right)-39.15 ; \\
K_{\text {lar }}=-133.3\left(d_{1} / d_{2}\right)+121.6 ; \\
K_{\text {small.pr }}=22.744 ; \\
K_{\text {lar.pr }}=22.087,
\end{gathered}
$$

where $K_{\text {small }}$ - value of small fraction; $K_{\text {med }}$ - value of average fraction; $K_{\text {lar }}$ - value of large fraction; $K_{\text {med.pr }}$ - the average preset value of the average fractions number; $K_{\text {lar.pr }}-$ the average preset value of the large fractions number and $K_{\text {small.pr }}-$ the average preset value of the small fraction number.

Consider a mathematical model 2. Based on the results of the nonlinear correlation the analysis dependence between the average factions' number and the diameter of the elliptical form cavity compensatory, we construct a mathematical model for two variables $\left(d_{1}, d_{2}\right)$, transforming it using the least squares method to obtain the analytical expression $K_{\text {med }}\left(d_{1}, d_{2}\right)$ in the form:

$$
K_{\text {med }}\left(d_{1}, d_{2}\right)=-25.651+2.436 d_{1}+0.01 d_{2}+0.04 d_{1} d_{2}-0.027 d_{1}^{2}-0.03 d_{2}^{2}
$$

Then, taking into account limitations in the range of variation compensation cavity axis and the number of both large and small fractions mathematical model is:

$$
K_{\text {med }}\left(d_{1}, d_{2}\right)=-25.651+2.436 d_{1}+0.01 d_{2}+0.04 d_{1} d_{2}-0.027 d_{1}^{2}-0.03 d_{2}^{2} \rightarrow \max
$$

under conditions $50 \leq d_{1} \leq 80,60 \leq d_{2} \leq 100,\left(d_{1} / d_{2}\right)<1$ :

$$
\begin{gathered}
K_{\text {med }}+K_{\text {lar }}+K_{\text {small }}=100 \\
K_{\text {small }} \leq K_{\text {small.pr }}
\end{gathered}
$$

Then:

$$
\begin{gathered}
K_{\text {small }}=126.3\left(d_{1} / d_{2}\right)-39.15 \\
K_{\text {lar }}=-133.3\left(d_{1} / d_{2}\right)+121.6 \\
K_{\text {med.pr }}=22.744 \\
K_{\text {small.pr }}=55.169
\end{gathered}
$$


Similar models are built to charge variable section. The results of the calculations performed to optimize the size of the compensation cavities that maximize the number of middle fractions. The calculation results for the two types of mathematical models chosen to charge different designs are summarized in Table 5.

Table 5. Results of the calculations selection and justification optimal balance compensation cavity axis elliptical shape.

\begin{tabular}{|l|c|c|c|c|}
\hline \multirow{2}{*}{ Charge design } & \multicolumn{2}{|c|}{ Axis ratio $d_{1} / d_{2}$} & Number of middle fractions, \% \\
\cline { 2 - 5 } & Model 1 & Model 2 & Model 1 & Model 2 \\
\hline Permanent section & 0.75 & 0.747 & 23.14 & 22.74 \\
\hline $\begin{array}{l}\text { With boiler expansion } \\
\text { in the end of the hole }\end{array}$ & 0.748 & 0.746 & 21.40 & 21.90 \\
\hline $\begin{array}{l}\text { With a spherical } \\
\text { insertion at the end of } \\
\text { the hole }\end{array}$ & 0.751 & 0.752 & 23.17 & 23.41 \\
\hline $\begin{array}{l}\text { With uniformly } \\
\text { arranged in spherical } \\
\text { column inserts }\end{array}$ & 0.75 & 0.748 & 26.71 & 26.49 \\
\hline
\end{tabular}

An analysis of the mathematical modeling of crushing models results for optimizing the borehole charge construction and the parameters of the compensatory elliptic form cavity showed that the number of large and small fractions should not exceed their average values $(22.0 \%$ and $55.169 \%)$ and, respectively, the average fractions - not less than $23.14 \%$. Therefore, the number of middle fractions will be maximized by changing the aspect ratio of the major and minor axes of ellipses (models 1 and 2) compensation cavity provided that small ellipse axis ranges in $[60 ; 80] \mathrm{mm}$, and large $-[60 ; 100] \mathrm{mm}$. Thus, with the maximum dimensions of the ellipse compensation cavity, its contour will be closely spaced to charges and the number of small fractions will increase. With the minimum axes of the ellipse will observe the large size fractions. The decision optimization models grounded rational dimensions ellipse axis compensation cavity, namely lower axle was $\sim 60 \mathrm{~mm}$ and larger $-\sim 80 \mathrm{~mm}$. At the same time, the charge with spherical inserts, which are evenly spaced along the column, is chosen as the most efficient charge for the average fractions ( $26.71 \%$, Table 5$)$. The obtained results of calculations for two models are close and well agree with experimental data, but at the same time model 2 provides high accuracy and visibility when substantiating the parameters of drilling and blasting operations.

\section{Conclusions}

1. For the purpose of conducting experimental investigations of the destruction of hard rock by charges of various designs explosive on the models, a method and methodology of simulation was developed.

2. According to the results of researches, the features of hard media destruction by charges of various designs explosives have been revealed, depending on the parameters of the elliptic-shaped form-compensating cavity.

3. The dependences of the granulometric composition distribution of model part which was detonated by charges of different deigns explosives when they were undermined on the compensating cavity were constructed. Established distribution uniformity of grain size to charge variable section compared to other designs charges. At the same time, the yield of both small and large fractions is evenly distributed, the average piece and the mass of the 
part reflected in the model of the model for charges of a variable section increases.

4. Completed mathematical modeling choices and justify rational structures of explosives on the results of experimental studies on hard media destruction compensatory cavity elliptical shape. According to the results of the regression-correlation analysis of particle size distribution of the explosion shattered, the model developed two mathematical models to optimize the parameters of the compensation cavity for maximum yield criterion medium factions.

5. The regularities of influence design explosive charge and the compensation cavity parameters on grain size distribution of hard media which destroyed by explosion were established. It is proved that the absolute value of the correlation coefficient of large and small fractions destroyed by the explosion of hard medium tends to 1 . These coefficients are interconnected by a stable linear dependence. For medium fractions, the number of which is insignificant, their correlation ratio is small and is nonlinear in relation to the dimensions of the compensating cavity.

6. The conducted studies allowed substantiating the parameters of the compensating cavity (the axis of small and large ellipses) in the rocks and the choice of the effective design of the charge of the explosive.

\section{References}

1. Ishchenko, K.S., Konoval, V.M. (2006). Improving the efficiency of explosive excavation workings in ore mines, Metallurgical and Mining Industry, 6, 68-70

2. Ishchenko, K.S., Ishchenko, O.K. (2009). Improving the efficiency of methods for controlling the explosive destruction of strong stressed rocks in deep mines, Coal, 2, 912

3. Kurinnyi, V.P. (2018). Theoretical foundations of explosive destruction of rocks. Dnipro: Economy

4. Efremov, E.I. (1987). The destruction of rocks by explosion energy. Kyiv: Naukova Dumka

5. Ishchenko, K.S., Kratkovskii, I.L. (2008). Experimental studies of the influence of the microstructure on the nature of the explosive destruction of the uranium ores of the Vatutinsky deposit, Bulletin of the State HEI "NMU", 8, 58-64

6. Efremov, E.I., Kharitonov, V.N., Semeniuk, I.A. (1979). Explosive destruction of outburst rock in deep mines. Moskva: Nedra

7. Kharitonov, V.N., Semeniuk, I.A., Chernugina, L.F. (1982). Controlled destruction of stressed and outburst rocks. Kyiv: Naukova Dumka

8. Bulat, A.F., Ishchenko, K.S., Osinnii, V.Ya. (2007). Method of conducting mining. Patent No 79129, Ukraine

9. Bulat, A.F., Ishchenko, K.S., Osinnii, V.Ya. (2009). Method of conducting mining. Patent No 88825, Ukraine

10. Bulat, A.F., Ishchenko, K.S., Osinnii, V.Ya. (2009). Method of formation of the cranial cavity in strong stressed rocks. Patent No 88827, Ukraine

11. Ishchenko, K.S. (2014). Method of simulation of explosive destruction of rocks. Patent No 104707, Ukraine

12. GOST 21153.0-75. Rocks. Sampling and general methods of physical testing. It was introduced on 01.07.75. Moscow: Publishing Standards, 1976

13. GOST 10181.2-81. Concrete mixtures. Methods for determining the density. It was introduced on 01/01/81. Moscow: Publishing Standards, 1981

14. GOST 21153.2-84. Rocks. Method for determining the ultimate strength under uniaxial compression. It was introduced on 01.07.86. Moscow: Publishing Standards, 1986

15. Temporary methodological instructions for the rapid determination of rocks the elastic 
properties by ultrasonic method on untreated core samples of geological exploration holes, (1987). Leningrad: VNIMI

16. GOST 21153.7-75. Rocks. Method for propagation speeds of elastic longitudinal and transverse waves. It was introduced on 01.01.75. Moscow: Publishing Standards, 1976

17. Efremov, E.I., Ishchenko, K.S., Nikiforova, V.O. (2014). Blast blend. Patent No 88299 , Ukraine

18. Ishchenko, K.S., Ishchenko, O.K. (2012). Explosive device condenser. Patent No 98546, Ukraine

19. Baron, L.I. (1960). Lumpiness and its measurement methods. Moscow: Publisher AN USSR

20. Ishchenko, K.S., Us, S.A., Vdovichenko, M.M. (2011). Solution of optimization models of choice and substantiation of parameters of drilling and blasting works for efficient destruction of anisotropic rocks, Geotechnical mechanics, 94, 272-282 